\title{
Measuring the Fractal Dimensions of Fume Aerosols in situ Using Light Scattering $\dagger$
}

\author{
I. Colbeck \\ Institute of Aerosol Science, University of Essex, ${ }^{*}$
}

\section{Introduction}

Many workers have found it convenient to work with what is defined as the boundary fractal of the agglomerate, obtained from the two dimensional projection of the structure. Others have studied the internal structure of agglomerates and obtained the mass or density fractal dimension. The boundary fractal dimension is generally less than the density fractal dimension as information is lost in the two dimensional image of a three dimensional object. In many studies rather drastic sample preparation techniques have been employed, starting with collection on a filter, and then perhaps resuspension in a liquid. The important structural information in delicate aggregates may not survive such preparations undistorted. Such sample preparation artifacts may be eliminated with in situ measurements. Numerous methods have been reported in the literature for colloidal particles (e.g. Raper and Amal, 1993) and aerosols which are then suspended in solution (Martin et al, 1986, Hurd et al., 1987); fewer have appeared for aerosols, in situ and we will only consider the latter.

\section{Scattering methods}

In situ optical techniques are highly desirable because of their ability to provide remote sensing in hostile environments. The amount of information regarding details of the structure of the particle clusters obtainable through optical techniques is significantly lacking compared with the straight forward concept of collection and visual examination by electron microscope. Both theoretical (Mountain and Mulholland, 1988) and experimental (Zhang et al., 1988, Gangopadhyay et al., 1991; Bonczyk and Hall, 1992) research has shown how it is possible to use light scattering to measure the mean size and fractal dimension. In systems without distinct surfaces scattering occurs from the bulk of the object and the fractal dimension relates mass, $M$, to radius

${ }^{*}$ Colchester, C04 3SQ, UK.

$\dagger$ Received Sep. 20th 1994 of gyration, $R_{g}$, by $M R_{g}^{D}$. Objects obeying this relationship are mass fractals such as those formed by diffusion limited aggregation.

The principle of a scattering experiment is shown in figure 1. In a scattering experiment, a beam of light is directed onto a sample and the scattered intensity is measured as a function of scattering angle $\theta$, to the incident direction. If the scattering object is a fractal of fractal dimension $D$ then the intensity as a function of angle is given by $S(q) \sim$ $q^{-D}$ where $S(q)$ is the scattered intensity (often called the structure factor) and $q$ is the scattered wave vector, a wavelength $\lambda, q=4 \pi \lambda^{-1} \sin \theta / 2$. This result is obtained under the approximation that multiple scattering with the aggregate is small, which is a good approximation for soot when the monomer size is small. This relation holds for $q R_{g}>1$, but $S(q)$ is constant for $q R_{g}<1$, and this crossover can be used to determine $R g$, the radius of gyration. Since length scales in typical colloids or aerosols range from $10 \mathrm{~nm}$ for the monomer size to hundreds of nanometers for the cluster size, scattering of light is often used.

It is now possible by comparison of the well established scattering-extinction measurement of the mean size to cluster radius of gyration to yield the fractal dimension, the mean radius of its monomers and the mean number of monomers per cluster (Sorensen et al., 1992). This method is of use when $q R_{g}$ is small and structure-factor measurements are inaccurate.

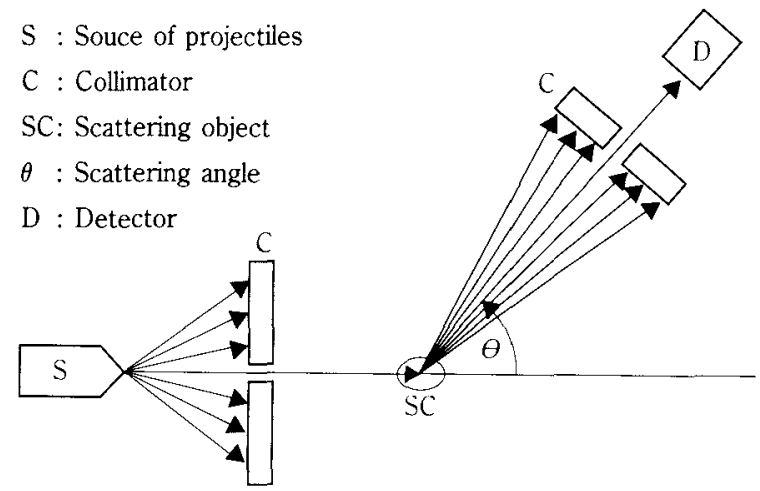

Fig. 1 The principle of a scattering experiment 


\section{Aerodynamic/mobility methods}

Schmidt-Ott (1988) reported on a method which involves comparing the aerodynamic radii of the agglomerated particle before and after tempering. Tempering causes the agglomerate to collapse into close-packed clusters of $D=3$. This technique is only valid for $D>2$ in the free molecular regime. Kutz and Schmidt-Ott (1990) have described a method based on a comparison of the mobility equivalent radius, determined by differential mobility analysis, with the ratio of mass and drag, measured by a low pressure impactor. For silver agglomerates produced by heating silver up to $1000^{\circ} \mathrm{C}$ they found a fractal dimension of 2.06 .

For one cluster falling with velocity $v_{1}$ and colliding with another moving with velocity $v_{2}$ the velocity of the resultant particle, $v_{3}$ is given by $v_{1}^{\gamma}+v_{2}^{\gamma}=$ $v_{3}^{\gamma}$ where $\gamma=D /(D-1)$ (Zhangfa et al., 1994). Hence if measurements of $v_{1}, v_{2}$ and $v_{3}$ are made the fractal dimension may be obtained. To observe the process of kinematic coagulation and to measure the settling velocities a CCD camera with output to a video-recorder was used (Figure 2). The process of kinematic coagulation could then be traced and photographed from the recorded images. The fractal dimension for carbonaceous particles ranged from 1.61 to 2.38 with an average value of 1.97. For magnesium oxide the fractal dimension ranged from 1.10 to 1.41 with an average value of 1.19 .

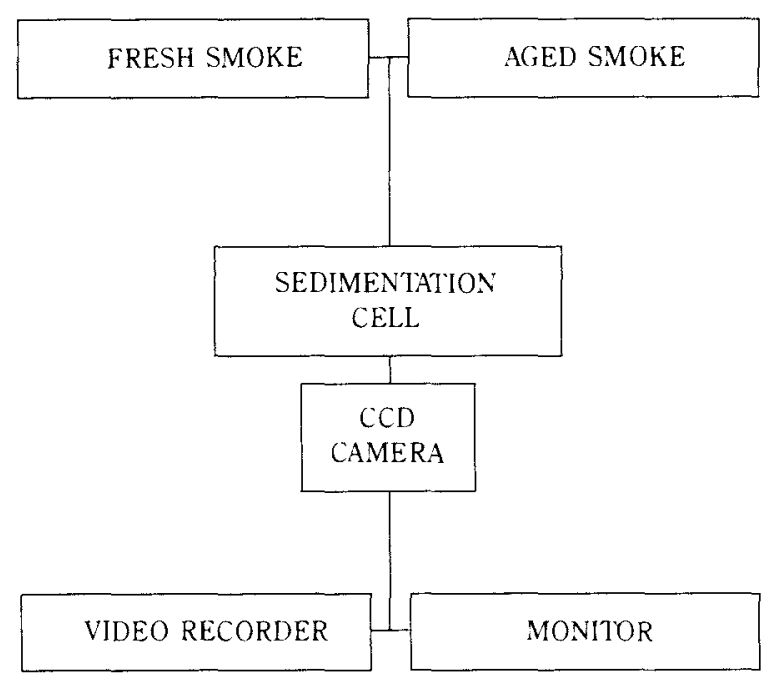

Fig.2 Schematic diagram of experimental apparatus for kinematic coagulation

\section{Other techniques}

By combining Inductively Coupled Plasma Optical Emission Spectrometry (ICP-0ES) with an epiphaniometer and Differential Mobility Analyzer it is possible to determine, simultaneously, the mass, surface area and diameter of agglomerated aerosols (Weber, 1992). From these measurements the fractal dimension and the monomer size may be determined. The results indicate that silver agglomerates, produced by spark discharge, are multifractal. In the free molecular regime the fractal dimension was 3 , while in the transition regime a value of 1.9 was obtained.

The fractal dimension has also been determined from measurements of the terminal velocity, $V_{T S}$, of a cluster and the electric field strength, $E$, required to balance the gravitational force (Colbeck et al., 1989). This technique utilises the classical Millikan cell. A plot of $\log V_{T S}$ against $\log E$ enables $D$ to be computed from the gradient. This method assumes that the clusters are spherically symmetric and they possess the same number of charges. These problems have been overcome by using a similar technique in conjunction with a photoemission method which enables the fractal dimension for single agglomerates in three dimensions to be determined (Nyeki and Colbeck, 1994). In this method a modified Millikan cell is used to obtain in situ measurements of $D$ for single agglomerates in the transition/ continuum regime (Colbeck et al., 1992). The method relies on the measurement of the aerodynamic diameter, based on a sedimentation technique, and the absolute mass of the agglomerate using a photoemission method. Hence the volume equivalent diameter $\left(d_{V}\right)$ and the measured aerodynamic diameter $\left(d_{a}\right)$ allow the dynamic shape factor and the mobility equivalent diameter $\left(d_{m}\right)$ to be calculated. The latter diameter is equivalent to the hydrodynamic diameter. The relationship between $d_{a}$ and the radius of gyration has been investigated both theoretically and experimentally and may then be used to derive the fractal dimension. The only parameters required for these determinations are an accurate estimation of the sedimentation velocity and various balance voltages in the cell (figure 3 ).

Measurements were reported for both soot agglomerates, up to $d_{a}=3 \mu \mathrm{m}$, and PVT spheres. The results for 10 soot and 4 PVT particles appear in Tables 1 and 2 respectively. The particle mass was calculated using a linear least-squares fit to the photoemission data and gave regression coefficients greater than $98 \%$ in all cases. From this calculation 


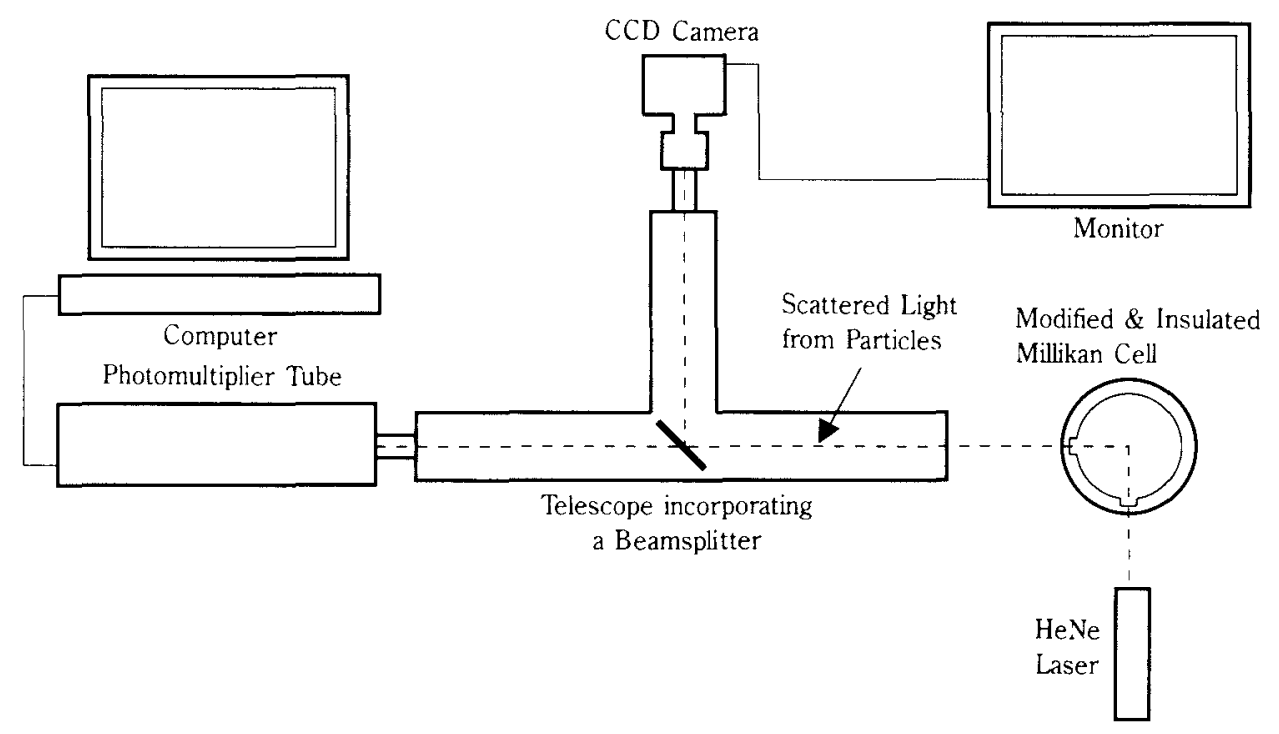

Fig.3 Schematic of the Modified Millikan Cell to determine the Fractal Dimension of Carbonaceous Agglomerates

Table 1 Data obtained via the Millikan cell method.

\begin{tabular}{|c|c|c|c|c|c|c|c|c|}
\hline Number & $\begin{array}{c}\text { Initial } \\
\text { Number } \\
\text { of charge }\end{array}$ & $\begin{array}{c}\text { mass } \\
(\mathrm{pg})\end{array}$ & $\begin{array}{c}d_{v} \\
(\mu \mathrm{m})\end{array}$ & $\begin{array}{c}V_{T S} \\
(\mathrm{~cm} / \mathrm{s})\end{array}$ & $\begin{array}{c}V_{T E} \\
(\mathrm{~cm} / \mathrm{s})\end{array}$ & $\begin{array}{c}d_{\mathbf{a}} \\
(\mu \mathrm{m})\end{array}$ & $\begin{array}{c}d_{m} \\
(\mu \mathrm{m})\end{array}$ & $\begin{array}{c}\text { Fractal } \\
\text { dimension }\end{array}$ \\
\hline 1 & 23 & 3.32 & 1.47 & 0.0035 & 0.0057 & 1.00 & 5.64 & 2.11 \\
2 & 15 & 3.48 & 1.49 & 0.0023 & 0.0028 & 0.80 & 8.83 & 1.93 \\
3 & 40 & 3.55 & 1.50 & 0.0029 & 0.0036 & 0.90 & 7.20 & 2.02 \\
4 & 12 & 1.13 & 1.03 & 0.0010 & 0.0015 & 0.51 & 6.36 & 1.83 \\
5 & 30 & 3.94 & 1.56 & 0.0022 & 0.0045 & 0.77 & 10.65 & 1.89 \\
6 & 27 & 3.25 & 1.46 & 0.0032 & 0.0047 & 0.94 & 6.08 & 2.07 \\
7 & 39 & 2.19 & 1.28 & 0.0031 & 0.0054 & 0.93 & 4.24 & 2.16 \\
8 & 39 & 11.30 & 2.21 & 0.0032 & 0.0074 & 0.95 & 20.68 & 1.85 \\
9 & 50 & 12.53 & 2.29 & 0.0048 & 0.0065 & 1.19 & 15.07 & 1.98 \\
10 & 10 & 4.14 & 1.58 & 0.0030 & 0.0031 & 0.91 & 8.23 & 1.99 \\
\hline
\end{tabular}

Table 2 Data for PVT spheres.

\begin{tabular}{|c|c|c|c|}
\hline $\begin{array}{c}\text { mass } \\
(\mathrm{pg})\end{array}$ & $\begin{array}{c}\text { Number } \\
\text { of charges }\end{array}$ & $\begin{array}{c}d_{a} \\
(\mu \mathrm{m})\end{array}$ & $D$ \\
\hline 4.67 & 14 & 2.06 & $2.99 \pm 0.03$ \\
\hline 4.63 & 28 & 2.07 & $2.99 \pm 0.03$ \\
\hline 4.71 & 23 & 2.03 & $2.96 \pm 0.03$ \\
\hline 4.95 & 39 & 2.07 & $2.96 \pm 0.03$ \\
\hline
\end{tabular}

the initial charge on the particle was found. In the final column of Table 1 the fractal dimension has been calculated with a primary spherule diameter of $50 \mathrm{~nm}$, measured from SEM images. Most values lie around $D=1.9$ for diffusion limited clustercluster (DLCC) growth but as they occur in the upper part of the range linear trajectories are implied (Meakin 1984). Such linear impacts may also occur due to differential coagulation, in which agglomerates collide with each other under gravitational sedimentation.

A number of agglomerates appeared with $D>2$ for which it was conjectured that restructuring effects were responsible (Nyeki and Colbeck, 1994: Nyeki and Colbeck, 1993). To illustrate the case two soot particles $(D=2.11$ and 2.16) were deliberately restructured by reversing the electric field polarity several times and observing the changing morphology on the monitor. In this manner an open structure could be transformed into essentially a spherical structure with ease. It is therefore possible that this mechanism was responsible for the other high observed values of $D$. Various models have been reported which simulate restructuring and readjusting 
effects present in real aggregation experiments. A simple model investigated by Jullien and Meakin (1989) allowed restructuring immediately after two agglomerates came into contact. For DLCC aggregation $D$ increased from $\sim 1.8$ to $\sim 2.1$ and reached $\sim 2.2$ after the last stage of restructuring. It was found that as restructuring occurred it became increasingly difficult for $D$ to increase. Thus in a similar manner reaction limited cluster-cluster (RLCC) aggregation only reached $D \sim 2.25$ as the initial value was relatively higher at $D \sim 2.0$. Although electrical restructuring mechanisms were not considered it is seen that simple restructuring mechanisms lead to increases in $D$. Important areas for the implications of agglomerate re-structuring, identified by Jullien and Meakin (1989) were rheology, and air and water pollution. Using the method presented here $D$ may be measured for a variety of materials and the effects of restructuring on agglomerates may also be observed.

As a verification of the technique and equations utilised PVT particles were used as calibration stan- dards. Fractal dimensions vary from $2.96-2.99$ and show excellent agreement to the theoretical value of 3 , hence verifying the method.

\section{Conclusions}

The characterisation of particle shape has been an active research area and source of contention for over fifty years. The application of fractal geometry to aerosol science lies in the necessity to parameterise complex aerosol shapes, such as those produced by combustion processes. Various in situ techniques have been presented allowing the fractal dimension of both a single agglomerate and an ensemble to be calculated. These methods are summarized in Table 3.

The instrument developed to study single particles has many applications. The ability to measure and monitor $D$ for single particles allows their microchemical/physical properties to be investigated. Diagnostic information on formation dynamics of aerosol agglomerates is embedded in the fractal structure. This is an area which will require further investigation.

Table 3 Summary of in situ techniques used to measure the fractal dimension of aerosols

\begin{tabular}{|l|l|l|l|}
\hline Author & Method & Material & $\begin{array}{l}\text { Fractal } \\
\text { Dimension }\end{array}$ \\
\hline Schmidt-Ott (1988) & mobility & silver & 2.18 \\
\hline Hurd and Flower (1988) & light scattering & silica & $1.49 \pm 0.15$ \\
\hline Zhang et al. (1988) & light scattering & soot from premixed $\mathrm{CH}_{4} / \mathrm{O}_{2}$ flame & $1.62 \pm 0.06$ \\
\hline Colbeck et al. (1989) & Millikan cell & soot from $\mathrm{C}_{4} / \mathrm{H}_{10}$ flame & 1.9 \\
\hline Kutz and Schmidt-Ott (1990) & aerodynamic & silver & 2.06 \\
\hline Gangopadhyay et al.. (1991) & light scattering & soot from premixed $\mathrm{CH}_{4} / \mathrm{O}_{2}$ flame & $1.6 \pm 0.15$ \\
\hline Bonczyk and Hall (1992) & light scattering & soot from $\mathrm{C}_{4} / \mathrm{H}_{10}$ flame & 1.49 \\
\hline Sorensen et.al. (1992) & light scattering & soot from premixed $\mathrm{CH}_{4} / \mathrm{O}_{4}$ flame & 1.73 \\
\hline Weber (1992) & ICP-OES & silver & $\begin{array}{l}3.0 \\
\text { free molecular } \\
\text { regime } \\
1.9 \\
\text { transition } \\
\text { regime }\end{array}$ \\
\hline Nheki and Colbeck (1994) & Millikan cell & & $1.87-2.19$ \\
\hline Zhangfa et.al. (1994) & aerodynamic & $\begin{array}{l}\text { soot from } \mathrm{C}_{4} \mathrm{H}_{10} \text { flame } \\
\text { magnesium oxide }\end{array}$ & $\begin{array}{l}1.97 \pm 0.07 \\
1.19 \pm 0.02\end{array}$ \\
\hline
\end{tabular}

\section{References}

1) Bonczyk P.A. and Hall R.J. (1992) Measurement of the fractal dimension of soot using uv laser radiation.Langmuir, 8, 1666-1670.

2) Colbeck I., Eleftheriadis K. and Simons S. (1989) The dynamics and structure of smoke aerosols. J. Aerosol Sci., 20, 875-878.

3) Colbeck I., Nyeki S. and Zhangfa Wu (1992) In situ measurement of the fractal dimension of
aerosols.J. Aerosol Sci., 23, S365-S368.

4) Colbeck I. and Nyeki S. (1993) On the measurement of the fractal dimension of individual restructured agglomerates. Aerosols: Their Generation, Behaviour and Application, The Aerosol Society, 123-127.

5) Gangopadhyay S., Elminyawi I. and Sorensen C.M. (1991) Optical structure factor measurements of soot particles in a premixed flame. Appl. Opt., 30, 4859-4864. 
6) oflund A.J., Schaefer D.W. and Martin J.E. (1987) Surface and mass fractals in vapor-phase aggregates. Phys. Review A., 35, 2361-2364.

7) Jullien R. and Meakin P. (1989) Simpel models for the re-structuring of $3 \mathrm{D}$ ballistic aggregates, J. Colloid Inter. Sci., 127, 265-272.

8) Kutz S. and Schmidt-Ott A. (1990) Use of a lowpressure impactor for fractal analysis of submicron particles.J. Aerosol Sci., 21, S47-S50.

9) Martin J.E., Schaefer D.W. and Hurd A.J. (1986) Fractal geometry of vapor-phase aggregates. Phys. Review A, 33, 3540-3543.

10) Meakin P. (1984) Effects of cluster trajectories on cluster-cluster aggregation: A comparison of linear and Brownian trajectories in two and three-dimensional simulations. Phys. Review A., 29, 997-999.

11) Mountain R.D. and Mulholland D.W. (1988) Light scattering from simulated smoke agglomerates. angmuir, 4, 1321-1326.

12) Nyeki S. and Colbeck I. (1994) The measurement of the fractal dimension of individual in-situ soot agglomerates using a Millikan cell technique. J. Aerosol Sci., 25, 75-90.

13) Nyeki S. and Colbeck I. (1993) Electric field induced restructuring of single, in-situ, carbonaceous aerosols. J. Aerosol Sci., 24, S547-S548.

14) Raper J.A. and Amal R. (1993) Measurement of aggregate fractal dimensions using static light scattering. Part. Part. Syst. Charact., 10, 239245.

15) Schmidt-Ott A. (1988) In situ measurement of the fractal dimensionality of ultrafine aerosol particles. Appl. Phy. Lett., 52, 954-956.

16) Sorensen C.M., Cai J. and Lu N. (1992) Light scattering measurements of monomer size, monomers per aggregate, and fractal dimension for soot aggregates in flames. Appl. Opt., 31, 65476557.

17) Weber A.P. (1992) Characterization of the geometrical properties of agglomerated aerosol particles. Ph.D. Thesis, Paul Scherrer Institut, Villigen, Switzerland.

18) Zhang H.X., Sorensen C.M., Ratmer E.R., Olivier B.J. and Merklin J.F. (1988) In situ optical structure factor measurements of an aggregating soot aerosol.Langmuir, 4, 867-871.

19) Zhangfa Wu, Colbeck I. and Simons S. (1994) Kinematic coagulation, aerosol agglomerates and the fractal dimension. Aerosols: Their Generation, Behaviour and Application, The Aerosol Society, 24-28.

\section{Author's short biography}

\section{Dr. Ian Colbeck}

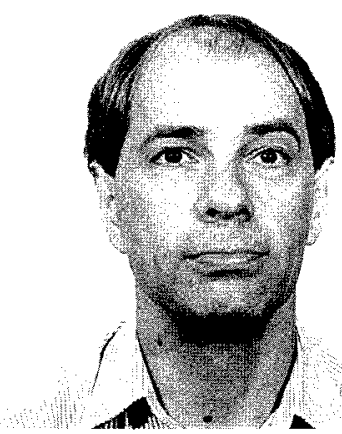

Dr. Ian Colbeck studied physics at Queen Mary College, University of London before obtaining an MSc in Atmospheric Physics at Oxford University and a $\mathrm{PhD}$ at Lancaster University in Environmental Physics. Initially appointed to teach a MSc course in Aerosol science and Technology he is currently a Senior Lecturer in the Department of Chemistry and biological Chemistry and Director of the Institute For Environmental Research, University of Essex. His fields of interest include photochemical oxidants, atmospheric modelling, the physicochemical properties of aerosols, filtration, characterization of particle shape and vapor-phase synthesis and processing of nanoparticle materials. 